\title{
Modern Modal Testing: A Cautionary Tale
}

\author{
James C. Akers, Kim D. Otten, Joel W. Sills ${ }^{1}$, and Curtis E. Larsen ${ }^{2}$ \\ NASA Glenn Research Center \\ 21000 Brookpark Road \\ Cleveland, $\mathrm{OH} 44135$ \\ ${ }^{1}$ NASA Johnson Space Center \\ 2101 NASA Parkway \\ Houston, TX 77058 \\ ${ }^{2}$ Texas Christian University \\ Department of Engineering \\ 2800 S. University Dr \\ Fort Worth, TX 76129
}

\begin{abstract}
Over the past 50 years, great advances have happened in both analytical modal analysis (i.e., finite element models and analysis) and experimental modal analysis (i.e., modal testing) in aerospace and other fields. With the advent of more powerful computers, higher performance instrumentation and data acquisition systems, and powerful linear modal extraction tools, today's analysts and test engineers have a breadth and depth of technical resources only dreamed of by our predecessors. However, some observed recent trends indicate that hard lessons learned are being forgotten or ignored, and possibly fundamental concepts are not being understood. These trends have the potential of leading to the degradation of the quality of and confidence in both analytical and test results. These trends are a making of our own doing, and directly related to having ever more powerful computers, programmatic budgetary pressures to limit analysis and testing, and technical capital loss due to the retirement of the senior demographic component of a bimodal workforce. This paper endeavors to highlight some of the most important lessons learned, common pitfalls to hopefully avoid, and potential steps that may be taken to help reverse this trend.
\end{abstract}

Keywords: Analytical Modal Analysis, Bimodal Workforce, Experimental Modal Analysis, Finite Element Analysis, Finite Element Model, Lessons Learned, Modal Testing, Retirement

\section{INTRODUCTION}

Analytical modal analysis (i.e., finite element modelling and analysis) and experimental modal analysis (i.e., modal testing) have advanced greatly over the past 50 years. Today's powerful computers, high performance instrumentation and data acquisition systems (DAQ's), and powerful linear modal extraction tools can provide today's modal test/analysis engineer the technical resources needed to competently tackle most, if not all, linear structural issues. However, even with this breadth and depth of technical resources, today's modal test/analysis engineer is still only as good as their technical expertise. For us "seasoned veterans" this technical expertise was gained during the course of 30+ years of working and study in the aerospace field and through the exceptional mentoring we received from many senior knowledgeable professionals. It was through this exceptional mentoring that we were able to leverage the expertise of the "seasoned veterans" that came before us and avoid making many poor (and quite frankly simple/unwise) mistakes. It is said "Good judgement comes from experience, and 
experience comes from bad judgement [1]." Unfortunately, or maybe fortunately, organizations do not have the luxury of being able to allow their engineers to make bad judgements. That said, recent trends observed in both individual engineers and in engineering organizations indicate the hard lessons learned over the past 50 years are being forgotten or ignored, and possibly fundamental concepts are not being understood. These troubling trends have the potential of leading to the degradation in the quality of and confidence in both analytical and test results and increase risk to the project. If this happens the credibility and efficacy of our engineering profession will come into question and missions may potentially suffer.

To some extent, these problems are a making of our own doing. With the advent of modern portable high power computers and user friendly automated software, it is relatively easy for a novice engineer to generate a computer aided-design (CAD) model of a structure, auto mesh it to generate a finite element model (FEM), and perform static and modal analysis (i.e., compute stresses, strains, modal frequencies, and mode shapes). Also a novice engineer can collect test data (i.e., force, acceleration, and strain time histories), post process it, and extract modal parameters (i.e., modal frequencies, mode shapes, and modal damping). In a work force where there are ample seasoned engineering professionals helping to guide and mentor less experienced novice engineers to avoid common pitfalls and reinforce the underlying engineering theoretical concepts, these modern capabilities are a true asset. Even in organizations that have a bimodal workforce where there are mostly very seasoned and very novice engineers, ample mentoring can help to make up for the shortfall of not having many, if any, midcareer engineers. However in organizations where the current and foreseeable programmatic budgetary pressures are limiting the amount of analysis and testing, combined with their technical capital loss due to the retirement of their senior seasoned professionals, which curtails much needed mentoring, the early career novice engineers are left "not knowing what they don't know". While the focus of this paper is on aerospace engineers performing modal/vibration testing or performing analysis with FEM's, the concerns discussed here apply to many engineering disciplines. The goal of this paper is to provide early career novice engineers with some key concepts to help avoid the most common pitfalls, and to provide both them and everyone in their organization potential steps for ensuring their organization's technical expertise remains viable and the aerospace engineering profession remains vibrant and relevant.

\section{PITFALL \#1: CONFUSING COMPUTER JOCKEYS AND ENGINEERS.}

As engineers we must understand the key fundamental physics based concepts that underpin our analysis and testing and realize that software and hardware are tools, not crutches to make up for our lack of understanding. We must be able to not only provide results, but be able to verify and validate those results. Being able to make "simple back of the envelope calculations" is of paramount importance to provide sanity checks. This is even more critical in today's work environment where engineers are increasingly operating as an "army of one" and the double checks and fact checking that occurred in the past are happening less and less frequently, if at all. If you can draw the free body diagram of a single degree of freedom (SDOF) oscillator, derive its equation of motion, solve for its impulse response function, solve for the frequency response function, be able to plot these functions and understand them, you are $90 \%$ of the way to understanding most vibration problems. The old adage "Keep It Simple and Straightforward" (KISS) is as relevant today as in the past. Start simply in your analysis/testing and only add complexity as needed. Don't confuse complexity for sophistication. Many times the most sophisticated solution method is the simplest. Remember, we went to the Moon on FEM's having only centerline grid points and beam elements, more commonly referred to as "ball-stick models." The Saturn V launch vehicle was initially modeled in this manner during the Apollo program [2,3]. Somehow million degree of freedom (DOF) FEM's were not required (and neither was the Internet).

\section{PITFALL \#2: TOO MUCH BLIND FAITH IN FEM'S.}

“All MODELS ARE WRONG BUT SOME ARE USEFUL [2].” This blind faith is the direct result of having extremely powerful computers allowing relatively novice engineers to generate $\mathrm{CAD}$ models that look exactly like the hardware and in turn using auto mesh features to generate FEM's that look exactly like the hardware. "Pretty pictures do not a good model make." This has led some decision makers to believe that accurate CAD models and FEM's can be accomplished with inexperienced (a.k.a. inexpensive) engineers. This is simply not true. A finely meshed FEM does not guarantee it accurately captures the structural characteristics (i.e., load paths) of the actual hardware. It only means the same modelling assumptions have been made many, many, many times and if these assumptions are incorrect then the resulting FEM will still be 
inaccurate. Also there is a current trend of using the stress FEM as the loads FEM. The stress FEM has very fine meshing to accurately capture the intricacies of stress fields around joints and fasteners. The loads FEM does not require as fine a meshing since it is intended to capture the structural dynamic properties and needs to allow reasonable modal analysis run times. The validity of CAD models and FEM's is highly dependent upon the proficiency and experience of the engineer generating them. The engineer generating the FEM needs to use the appropriate elements instead of simply relying on automeshing that picks element types based upon ease of meshing instead of the behavior of the underlying structure. Similarly, the FEM automatic single point constraint (AUTOSPC) feature should be avoided so the engineer is forced to understand what DOF are constrained and unconstrained in the FEM and why. Remember that until a FEM has been "grounded" to test data (i.e., correlated to static and modal test data), its accuracy/validity is uncertain. Incorporation of static test results into a FEM correlation to obtain the correct stiffness is half the battle - never forget the dynamic side of the equation (i.e., square root $(\mathrm{k} / \mathrm{m})$ ). For modal testing pretest analysis, this means having a healthy skepticism of the FEM accuracy and always incorporating margin into test preparations (e.g., skewing shakers to help ensure that all Target Modes will be adequately excited, instrumentation at the boundary to verify the boundary conditions during testing, using engineering judgement along with such tools as iterative kinetic energy to select instrumentation locations).

\section{PITFALL \#3: CONFUSING BEING BUSY WITH BEING PRODUCTIVE.}

Avoid confusing being busy with being productive. People naturally want to feel that they are making progress towards reaching their end goal and it is natural to assume that if they are busy doing something instead of being "idle", then they are surely making progress. Not true. Take the necessary time to plan, prioritize, and define the objectives, goals, and success criteria of the task. This will allow you to focus your time and resources to give you the highest probability of successfully completing your task. Note there are no certainties and you must realize that you do not control everything. Hence, we can only speak of probabilities and likelihoods of success.

\section{PITFALL \#4: AVOID RUSHING TO ANALYZE.}

It is very tempting when first given a model or data to want to jump right in and start performing your analysis. See Pitfall \#3. However, it is important to perform "sanity checks" on any models or data prior to performing any analysis in order to avoid working for weeks only to discover it was all for naught because of either a faulty model or corrupted data. Be skeptical of FEM's provided to you and perform your own standard model checks, especially if the previous FEM analysis resides in a different version of software than you are using because default settings can change [5]. Be sure the FEM has been validated to match the CAD or as-built hardware. A FEM is not valid just because it has been "correlated" to modal parameters extracted from a modal test or results from a static stiffness test. Taking the time to match the FEM to the CAD or as-built hardware is absolutely critical for accurately capturing the load paths and being able to predict internal loads [6]. Moreover, when the FEM matches the CAD or as-built hardware, $90 \%-95 \%$ of the model correlation effort is achieved. If the FEM does not accurately represent the CAD or as-built hardware, the model correlation effort, while being inaccurate, most likely will also be extremely difficult and time consuming and probably lead to unmet requirements and schedule constraints. Be sure you understand the units the FEM is in and the data units. Even though it is the $21^{\text {st }}$ century, many FEM's generated and used by US companies still have mass in units of slinches and slugs and acceleration data can be in units of in/sec 2 or g's. Don’t forget frequencies can be in units of $\mathrm{rad} / \mathrm{sec}$ or $\mathrm{Hz}$. When doing your checks, if you are off by a factor of 9.8, this is probably an acceleration units issue and if you are off by a factor of 6.3 (i.e., $2 \pi$ ), this is probably a frequency units issue.

Similarly be skeptical of data provided to you, and if possible, perform time-domain and frequency domain data quality checks as appropriate. Ask questions to the entity providing the data for poof of its validity. For example, if time history data has been supplied and has either been clipped or is in the noise floor of the data acquisition system that was used to record it, blindly post processing it into spectra will lead to very misleading spectra, which is not self-evident.

Take time throughout your analysis to perform intermediate checks. With today's software it is very easy to plot results and generate tabular listings, which provide quick and insightful checking methods. For example if you are filtering time histories, be sure to compare the frequency response function of the filter to the Power Spectral Density (PSD) computed on 
broadband random white noise that has been passed through it to verify the filter. It is very easy in to design filters that have very tight passbands, but are numerically unstable. It is of critical importance to be able to compute "back of the envelope" calculations for providing at least cursory sanity checks of analytical results. Some technical references to consider are listed in [7-24]. Documenting these intermediate and final checks constitute the important portion of your analysis report that verifies the validity of your analysis results. At the end of the day, it is your name and reputation that is on the line, and it only takes one or two bad analyses to give you a negative reputation in your organization, which can be very hard to overcome.

\section{PITFALL \#5: AVOID RUSHING TO TEST.}

Setting up a modal test and performing it, can be both a very exhilarating and stressful time. It involves physical exertion (e.g., moving shakers, mounting accelerometers, running cabling, being in an unfamiliar locale), upper management and programmatic personnel being present to "witness" the testing (i.e., looking over your shoulder, being in a fish bowl), and having a very limited time window to accomplish it. Because of this, it is very natural to want to start collecting data as quickly as possible, and in turn to start extracting modal parameters as quickly as possible. However, as with analysis, it is important to take time to verify the test setup and the validity of the data being collected. Be sure the DAQ has anti-aliasing filtering engaged and operating. With the advent of faster and faster computers, a common misconception going around is that you can sample so fast that aliasing is no longer an issue. Not true. Aliasing will always be present whatever the sampling rate is and once the recorded time histories have been corrupted by aliasing it is impossible to correct them. Take the time to perform time-domain and frequency-domain data quality checks during testing to verify the health of the channels and validity of the test setup.

Time domain checks should include visual inspection of the time histories, looking for data dropouts, digitization error (i.e., full scale setting of the DAQ set too high), clipping (i.e., full scale setting of the DAQ set too low), adequately capturing peaks in the frequency range of interest, and impulsive spikes indicating rattling and/or impacting taking place. Computing standard statistics such as mean, standard deviation, kurtosis, and crest factor on random excitation test runs provide a quick and useful check to determine if nonlinear behavior is present [25]. Keep in mind that crest factors (i.e., peak divided by standard deviation) during vibration testing can reach as high as 5 or more. However, extremely high crest factors and kurtosis may indicate impulsive spikes in the time histories of channels going bad or measuring significant rattling/impacting in the test article. It is important to monitor channels in real time as they are being acquired to check for underflow, overflow, excessive mean, signal drift, and impulsive spikes. If the DAQ generates an automatic channel fault log (i.e., dead channel, overflow, underflow, etc.) take advantage of this important feature.

Frequency domain checks should include verifying the drive point frequency response function (FRF) has the expected phase angle behavior (i.e., for a force to acceleration FRF if the accelerometer has the same orientation as the load cell the phase angle stays between $-180^{\circ}$ and $-360^{\circ}$ over the desired frequency range). Check the input excitation force PSD and compare the response PSD's to their ambient background levels to verify the excitation has sufficient frequency bandwidth and level. Check the coherence plots to verify there are linear relationships between input excitation forces and responses confirming the adequacy of the input excitation force level. Check the FRF for excessive hashiness, indicating either too low an input excitation force level and/or nonlinear behavior in the test article. Are the FRF resonance peaks symmetric and well-shaped or are they tipped to the right or left (i.e., "shark fin" pattern) that indicates nonlinear behavior in the test article? PSD or Fast Fourier Transform (FFT) waterfall plots (also called spectrograms) are a very useful tool for determining if test article is behaving nonlinearly or its structural dynamics are time varying, which may indicate degradation of its structural health (i.e., damage is occurring). Keep in mind that PSD or FFT waterfall plots computed on sine sweep data may have subharmonic or super harmonic components that are due to the nonlinear behavior of the input excitation. While electrodynamic shakers can be predominantly linear, as they are driven to higher force levels they will also become nonlinear due to large displacement of their alignment flexures. Hydraulic shakers, which have the advantage of being able to provide higher force levels at lower cost than electrodynamic shakers, typically have significantly higher nonlinear behavior than electrodynamic shakers given the same drive signal. However, pre-distorting a hydraulic shaker's drive signal can significantly reduce the amount of total harmonic distortion in the force it generates. 
Be sure to instrument the support structure the test article is mounted to in order to verify/measure the test boundary conditions. This is true for both modal and vibration testing. While floors, strongbacks, slip tables, and expander heads may be very stiff, they typically do exhibit some flexing or rigid body motion during testing. Without this boundary condition information, deflections in the support structure cannot be seen in the extracted test mode shapes and any subsequent FEM model correlation will erroneously attribute support structure flexibility/dynamics to the test article.

Acquiring ambient background levels at least at the beginning and end of each test day, and prior to each new test configuration (e.g., moving of shakers or accelerometers, reconfiguring the test article) is vital in being able to understand the ambient background level and to verify the health of the test setup. Having the ambient background level will also help determine minimum input excitation levels, which avoids wasting time performing modal testing with input excitation levels being too low. Keep in mind that if the response signals are in the noise floor, no amount of averaging is going to clean them up. Many times high ambient background levels are due to florescent lighting, heating, ventilation, and air conditioning (HVAC) running, and pumps and motors operating in adjacent rooms. Turning these off or testing when they are not in operation can significantly drop the ambient background levels. This is why many modal tests, since they need to measure very low level responses, are conducted during off-shift hours and/or on weekends.

Once a preliminary set of FRF have been computed, if possible extract an operating deflection shape at a frequency well below the lowest resonance peak frequency and animate this with your Test Display Model (TDM). This provides a quick visual check that the accelerometers have been setup correctly (i.e., location, orientation, sensitivity). It is strongly recommended that all of these checks be done at the start of the modal test to verify the validity of the entire test setup. If these checks are not first completed, typically what happens is that errors are discovered a few at a time over the course of several test runs. This in turn quickly leads to a bookkeeping headache of having to go back, if possible, and correct not only the original test data, but all of the post processed results generated from that corrupted test data (i.e., FRF, mode shapes, etc.).

Perform low level random or sine sweep characterization test runs before and after any high level modal or vibration testing to monitor the structural health of the test article. This is necessary in order to be able to determine if observed shifts in the FRF resonance peaks at different input excitation levels is due to a permanent change in the test article (e.g., damage, settling) or is due to nonlinear behavior (e.g., joints breaking free and slipping). These pre and post characterization test runs also answer the important question of whether the modal/vibration test affected the structural health/characteristics of the test article.

When performing a modal hammer survey, start with the softest hammer tip and only go onto stiffer/harder tips if needed to obtain the desired frequency bandwidth. Stiffer/harder tips excite higher frequencies and more strongly excite nonlinearities in the test article, which degrades the FRF, and produces responses with higher peak responses, which eats up dynamic range on the DAQ.

\section{PITFALL \#6: LACK OF DOCUMENTATION.}

For both the analyst and test engineer it is vital to keep a running log of your work. For the analyst this can be a running summary memo that documents your daily work, which should include the models used, the results generated, file locations, and important findings. For the test engineer this can be a test log, which includes the data acquisition and post processing parameters, channel table, test run log, and file locations. Photo document (including labels) as you go throughout the test. There is NO excuse with the proliferation of excellent digital cameras and cell phone cameras to NOT have an abundance of photographic evidence. Generating quick look memos/reports after each major testing phase helps to keep all stakeholders informed as to testing status/progress. Start writing the draft of the test report and test presentation as you go during the test so that key results, lessons learned, and key insights are captured. These drafts should be $90 \%$ complete by the end of the test. It is very difficult to remember details sometimes even days later, let alone weeks after a test has wrapped up. For both the analyst and test engineer, this documentation forms the foundation of your personal technical encyclopedia of technical knowledge and accomplishments, which you can draw and build upon as your career progresses. You typically will encounter many similar tasks throughout your career, and your technical encyclopedia will be an invaluable asset to you. 


\section{PITFALL \#7: DESIGNING ONLY FOR DESIGN LOADS.}

Hardware needs to be designed for all of the environments it will be exposed to, which includes not only design loads (i.e., maximum expected flight environment (MEFL)), but also testing loads, which in the case of vibration qualification testing may be MEFL + 3dB [26]. Ideally the associated upper test tolerance (e.g., for random vibration testing the acceleration PSD test tolerance is $+3 \mathrm{~dB}$ ) should also be included in the design so that if the achieved test level exceeds the nominal test level, which often occurs, testing does not need to be suspended while an analytical assessment of the hardware is made. In addition the hardware needs to be designed so that it can be mounted for testing, which means discussions with the test lab and the designer of the test fixturing. Many times the test fixture is nothing more than an adapter plate that goes from the bolt-hole pattern of the hardware to the bolt-hole pattern of the slip table/expander head of the facility shaker. For more complicated test fixturing, verify the test fixture does not introduce unwanted dynamics during testing (i.e., the integrated hardware and fixture have modes different from the fixed-base hardware over the test frequency range). Modes introduced by the test fixture have two detrimental effects. First, is that these modes may produce narrowband vibration environments that significantly exceed what the hardware can withstand. Second, is that the test fixture acts as a lowpass filter and attenuates the vibration environment the hardware sees above the frequencies of these modes and therefore leads to under testing. For force limited vibration testing, the hardware mounting tabs need to not only meet stiffness requirements, but also need to be of sufficient size to properly interface with the load cells used to measure the total amount of force going into the test article.

\section{PITFALL \#8: MODAL ANALYSIS NOT ACCOUNTING FOR OUT-OF-BAND DYNAMICS.}

Residual vectors, or equivalently a mode acceleration approach, need to be included in any analysis until they are shown to be unnecessary to account for structural dynamics that lie out-of-band the frequency band of interest. Not accounting for structural dynamics either below or above the frequency range of interest can lead to wildly inaccurate FEM results. In modal testing pretest analysis where initial shaker locations and orientations are selected, residual vectors are needed to account for potentially significant compliance in the test article at the shaker drive points. Not including residual vectors can erroneously lead the modal test engineer to over predict how well the target modes will be excited and therefore lead to poorly choosing the initial modal shaker locations and orientations, which in turn will lead to major changes in the shaker setups during the modal test and possibly not being able to meet schedule or excite all target modes.

If possible, consider the real environments used do derive the design loads. Do the real environments have significant frequency content outside the frequency range of the design loads? Is the type of design load conservative with respect to the time-domain characteristics of the real environments (i.e., is the environment random and stationary or is it transient)? Does the analysis produce results that are conservative enough, but not too conservative, to account for out-of-band energy and differences between the real environment type and that of the design loads?

\section{PITFALL \#9: ASKING FOR HELP OR SEEKING ADVICE IS A SIGN OF WEAKNESS.}

A common misconception is that asking for help or advice is a sign of weakness and incompetence. Not true. No one knows everything, especially newer and less experienced engineers. Even the more experienced "seasoned" engineers fall into this category, but they typically understand what questions to ask. Always understand that you "do not know what you don't know". Asking colleagues to look over your work or asking for advice is an expected and welcomed behavior in a healthy organization. Senior engineers want to share their experience, knowledge, and insights. Unfortunately, due to current and foreseeable budgetary pressures, novice engineers are many times not being paired directly with senior engineers leading to the novice engineers not having a naturally occurring "apprenticeship" period early in their careers. Therefore, unfortunately the burden falls on the novice engineers to actively seek out the advice of their senior colleagues. Apprenticeship is important and needs to once again become a norm in engineering. Make that connection!

\section{PITFALL \#10: STOVE PIPING: SEPARATING ANALYSTS AND TEST ENGINEERS.}

This is quite a common practice that leads to tension and potential disconnects between the analysis and testing groups/personnel, diminishes the technical prowess/capability of the organization, and adds risk to programs. Having 
analysts supporting testing provides them with the much needed access to the actual hardware and the ability to gain a deeper appreciation/understanding for the assumptions/simplifications they have incorporated into their FEM. It also provides analysts the opportunity to see firsthand the challenges the test engineer faces conducting vibration/modal testing (e.g., mounting instrumentation and shakers, obtaining valid/quality test data, extracting modal parameters, etc.) The analyst also gains insight into the fundamental structural dynamic characteristics of the hardware in its test configuration. Likewise, test engineers performing analysis gain an appreciation of the work that went into generating the FEM's, particularly the assumptions about joints and connections, which heavily influence the modal characteristics. The test engineers also gain insight into the challenges associated with the complexity and sophistication of the analytical work. I encourage you, if given the opportunity, to do both test and analysis work. It will only make you a more experienced and knowledgeable engineer and technically strengthen your organization.

Another related poor practice is not having interdisciplinary teams (i.e., "Tiger Teams") incorporated into programs, particularly at their start. Tiger Teams cover all aspects of a piece of hardware including: design, analysis, testing, operation, and decommissioning. This unfortunate practice is again partly due to current budgetary pressures and the misconception that Tiger Teams are more costly. While it is true that Tiger Teams may cost more during the initial phase of a program, they actually reduce the overall program cost by helping to ensure a good initial design and a well laid out plan for the subsequent analysis, testing, operation, and decommissioning are developed. Starting with a poor initial design can be very costly to remedy, especially the later in the program its shortfalls are identified. If you are presented with an opportunity to serve on a Tiger Team, do so! This will help you develop the ability to effectively communicate and work with other disciplines, which is critical to both a program's and organization's success.

\section{PITFALL \#11: TEST IS A FOUR LETTER WORD.}

Due to a combination of current budgetary pressures and decision makers becoming less technically knowledgeable, there is tremendous schedule pressure to reduce the amount of analysis and especially the amount of testing. A common misconception is that testing increases a program's cost, which is not accurate [27]. However, because a test has a welldefined resource allocation (i.e., cost, personnel, and schedule), this makes it very easy for decision makers to believe that cutting testing will save the program time and money. Unfortunately testing provides information about the hardware that is simply not available from unverified and unvalidated FEM's. Hence the residual risk in the FEM, which many times is not well understood, must be carried along and typically results in having to incorporating higher uncertainty factors. Many times these high uncertainty factors in turn lead to predicted negative structural margins, which leads to redesign or descoping the hardware's performance.

If possible, piggyback "mini-modal" hammer or base drive modal tests onto existing planned component and subassembly vibration tests to gain insight early in the program. Not only will this allow these FEM's to be test correlated, but it serves as a vital check on the design and identifies problems early when they are less costly and time consuming to fix. Always try to implement the philosophy of build from the foundation UP, not from the final configuration top DOWN. However, be careful of not falling prey to "tests of opportunity" and the "something is better than nothing" approach, where short cuts are taken due to lack of funding and schedule resulting in tests being poorly planned and executed. Decision makers may see "tests of opportunity" providing risk reduction to the program, when in reality their results may be at best confusing and most likely misleading.

Because of this current environment, engineers need to not only communicate to decision makers the who, what, when, and where, but most importantly be able to succinctly explain $\underline{\boldsymbol{w h} \boldsymbol{y}}$ the modal or vibration test is needed and the value they bring to the program in terms of reducing risk (i.e., increasing confidence the system will satisfactorily perform given the program's risk profile). Risk, schedule, and cost is the language of decision makers and engineers need to be comfortable and proficient in talking in these terms. Being able to succinctly convey essential concepts in easily understandable language is critical to the success of both you, your organization, and the project/program you are working on. Be able to advocate for the "right" test and to convey the associated risk with a "test of opportunity". Always have a "30 second elevator" speech in the back of your mind ready to go. You can be pulled into high level meetings at a moment's notice, and none of the attendees may have any technical knowledge in your area. 


\section{PITFALL \#12: UNREALISTIC SUCCESS CRITERIA.}

Whether it be for an analysis task or for a modal/vibration test, if at all possible define the success criteria at the very beginning and get agreement from all stakeholders. Along with the success criteria identify the receivables, task scope, task schedule (i.e., milestones), and the deliverables. For modal pretest analysis the success criteria includes proper selection of the Target Modes, excitation methods (i.e., shaker and hammer) and locations, test DOF set (i.e., ASET- NASTRAN analysis set of constrained boundary DOF) and the associated TDM, types of instrumentation, DAQ other computer resources, and the format the modal test results will be delivered to the customer. If modal shakers will be used, this also includes the coordination of how these shakers will be supported/suspended during the modal test, which many times is the most challenging aspect of any modal test. Always leave a little margin in the test schedule (i.e., success oriented) because no test goes exactly according to plan and tasks always take longer than expected. A too aggressive test schedule can result in needing to support long testing hours, which leads to increased stress, which leads to sleep deprivation, which leads to the test team making mistakes, which leads to testing delays, which leads back to long testing hours. Not a good situation.

Sometimes there really are no firm requirements. You have been called in by the Customer, who only knows that they have a problem, but they don't know the cause nor have they seen it before, but need it fixed rapidly. If possible acquire data during operation when the problem is occurring. Be mindful the cause of the problem may be spatially and/or temporally separated from the part that is failing. In the case of electronic components, even though failure occurred during a vibration fatigue test, the cause of the failure can be due damage incurred during the preceding thermal test [20,21].

Many times the Customer has a testing requirement levied upon them from a company or program, but they themselves do not fully understand nor appreciate the effort needed to meet this requirement. As the test engineer, you provide that vital role of "technical requirements translator" informing the Customer of the type and scope of testing that is required and how this fits into the overall test/analysis program requirements. Be sure to account for this in your work estimate, because this can take significant time (e.g., need to support review panels and technical meetings).

Modal testing extremely large hardware may also require a forced response analysis to ensure the selected excitation method and locations can produce response levels throughout the test article that are "significantly" above the ambient background. The ambient background is a combination of the ambient vibration levels in the test article, sensor and DAQ noise and signal drift, and the ambient electromagnetic environment. As stated earlier in Pitfall \#5, this may require performing the modal test during off-shift hours and/or on weekends. It may also require using a greater number of exciters (e.g., modal shakers), larger exciters, or instrumentation that is more sensitive. Understanding both the ambient background and the test article's modal damping characteristics are critical in this assessment.

In all pretest analysis, there needs to be a healthy dose of skepticism and some conservatism added because the pretest analysis is based upon an uncorrelated FEM and assuming perfect test boundary conditions. The selection of the test DOF needs to be done judiciously and sparingly because more instrumentation does not guarantee better self-orthogonality (ORTHO)/cross-orthogonality (XORTHO), in fact in many cases it causes significant degradation. Also more instrumentation requires longer installation and removal time, more effort during testing to keep them healthy and functioning, extra cost to purchase/rent the extra instrumentation and the associated DAQ channels, and incurs the extra burden of handling larger sized files. Always, as a final check of the selected test DOF, display the Target Modes (i.e., select modes of interest that contain the fundamental characteristics of the test article needed to achieve a well-correlated FEM) with the TDM. The TDM should only contain the grid points where instrumentation is installed. If the TDM display of the Target Modes does not make intuitive sense, then the test DOF set (i.e., ASET) needs to be further revised.

\section{CONCLUSION}

The advancements made in both analytical and experimental modal analysis in the aerospace field is beyond amazing. This is an exciting time for college students studying to go into and new engineers entering this field. This paper, by identifying twelve specific pitfalls, will hopefully serve as a guide to both groups to help ensure they have a successful career by pointing out some of the most common pitfalls and misconceptions. Well informed, their energy, enthusiasm, and drive will lead to the aerospace engineering profession remaining viable, vibrant, and relevant. 


\section{REFERENCES}

[1] Brown, Rita Mae “Alma Mater”, Random House Publishing Group, 2001.

[2] Grimes, P. J., McTigue, L. D., Riley, G. F., and Tilden, D. L., “Advancements in Structural Dynamic Technology Resulting from Saturn V Programs, NASA-CR-1539,Volume I, June 1970.

[3] Grimes, P. J., McTigue, L. D., Riley, G. F., and Tilden, D. L., "Advancements in Structural Dynamic Technology Resulting from Saturn V Programs, NASA-CR-1539, Volume II, June 1970.

[4] Box, George E.P. "Robustness in the strategy of scientific model building", Robustness in Statistics, Academic Press, 1979.

[5] Rose, Theodore, "Your Model Is Wrong", NESC Loads \& Dynamics Technical Discipline Team Face to Face, April 2018.

[6] Kabe, Alvar "Mode Survey Tests and Mode Shape Orthogonality Checks" NESC Loads \& Dynamics Technical Discipline Team Face to Face, April 2018.

[7] Avallone, Eugene A. and Baumeister III, Theodore "Marks' Standard Handbook for Mechanical Engineers", McGraw-Hill, $9^{\text {th }}$ ed., 1987.

[8] Bayer, William H. "CRC Standard Mathematical Tables", CRC Press, $24^{\text {th }}$ ed., 1974

[9] Beer, Ferdinand P. and Johnston Jr., E. Russell "Mechanics Of Materials", McGraw-Hill, 1981.

[10] Bendat, Julius S. and Piersol, Allan G. "Engineering Applications of Correlation and Spectral Analysis", John Wiley \& Sons, 1980.

[11] Bendat, Julius S. and Piersol, Allan G. "Random Data: Analysis and Measurement Procedures", John Wiley \& Sons, $2^{\text {nd }}$ ed., 1986.

[12] Blevins, Robert D. "Formulas For Natural Frequency And Mode Shape", Krieger Publishing, 1995.

[13] Craig Jr., Roy R. and Kurdila, Andrew J. "Fundamentals of Structural Dynamics", John Wiley \& Sons, $2^{\text {nd }}$ ed., 2006.

[14] Ewins, D.J. "Modal Testing: Theory, Practice and Application”, Research Studies Press LTD., $2^{\text {nd }}$ ed., 2000.

[15] Greenwood, Donald T. "Principles Of Dynamics", Prentice-Hall, 1965.

[16] Harris, Cyril M. "Shock and Vibration Handbook", McGraw-Hill, $3^{\text {rd }}$ ed., 1987.

[17] Horn, Roger A. and Johnson, Charles R. "Matrix Analysis", Cambridge University Press, 1991.

[18] McConnell, K.G. "Vibration Testing Theory and Practice", John Wiley \& Sons, 1995.

[19] Oppenheim, Alan V. and Willsky, Alan S. "Signals \& Systems", Prentice Hall, $2^{\text {nd }}$ ed, 1997.

[20] Steinberg, Dave S. "Vibration Analysis for Electronic Equipment”, John Wiley \& Sons, $3^{\text {rd }}$ ed., 2000.

[21] Steinberg, Dave S. "Preventing Thermal Cycling And Vibration Failures In Electronic Equipment", John Wiley \& Sons, 2001.

[22] University of Massachusetts Lowell "Modal Space", https://www.uml.edu/Research/SDASL/Education/ModalSpace.aspx.

[23] Wirsching, Paul R., Paez, Thomas L., and Ortiz, Keith "Random Vibrations: Theory and Practice”, John Wiley \& Sons, 1995.

[24] Young, Warren C. “Roark's Formulas for Stress and Strain”, McGraw-Hill, $6^{\text {th }}$ ed., 1989.

[25] Bendat, Julius S. "Nonlinear Systems Techniques and Applications", John Wiley \& Sons, 1998,

[26] NASA-STD-7001B "Payload Vibroacoustic Test Criteria", November 11, 2017.

[27] Juranek, Jeff B. and Wright, Charles P. "Improving Efficiency in Space Vehicle Assembly, integration, and Test (AI\&T)”, 30th Aerospace Testing Seminar, March 2017. 